\title{
FE-MAC: A Forwarding Election-based MAC Protocol for Wireless Sensor Networks
}

\author{
JianQiang \\ School of Computer, National \\ University of Defense Technology, \\ Changsha, 410073, China \\ +86-13786192176 \\ alloysteal@hotmail.com
}

\author{
Gong Zhenghu \\ School of Computer, National \\ University of Defense Technology, \\ Changsha, 410073, China \\ +86-731-4575980 \\ gzhu@nudt.edu.cn
}

\author{
Gui Chunmei \\ School of Computer, National \\ University of Defense Technology, \\ Changsha, 410073, China \\ $+86-13787081041$ \\ plantsperfume@yahoo.com
}

\begin{abstract}
Influenced by the design principles of the layered network protocol stack, current MAC protocols in wireless sensor network usually resort to improving energy efficiency as a major energy saving means, with little considerations of load balance, which limits the network lifetime. In this paper, a forwarding electionbased MAC protocol, FE-MAC, is proposed. By means of load balance improvement, energy is evenly consumed among nodes, and network lifetime is further extended. FE-MAC protocol adopts the forwarding election mechanism, in which nodes are organized to contend for the right of packet forwarding according to their residual energy, the more energy a node has, the more forwarding tasks it will take, and load balance and routing capability are realized. The experiments show that: compared with S-MAC and T-MAC, in wireless sensor networks of different scale, FE-MAC can prominently extend network lifetime, and the larger the number of nodes is, the better FE-MAC protocol performs. FE-MAC is more suitable to configuring large scale wireless sensor networks.
\end{abstract}

\section{Keywords}

Wireless Sensor Network, Medium Access Protocols, Energyefficient, Load Balance, Cross-layer Design, Trade-off.

\section{INTRODUCTION}

In wireless sensor network (WSN), media access control (MAC) protocol lies in the low layer of the protocol stack, which is crucial to guarantee WSN's efficient communication. The nodes in WSN usually have limited energy and it's battery is hard to be charged, therefore it becomes the first design objective for WSN MAC protocol to reasonably utilize energy and extend network lifetime $^{[1]}$.

Energy efficiency improvement and load balance are two effective means for WSN to utilize energy reasonably. Yet, according to the design principle of the traditional layered

Permission to make digital or hard copies of all or part of this work for personal or classroom use is granted without fee provided that copies are not made or distributed for profit or commercial advantage and that copies bear this notice and the full citation on the first page. To copy otherwise, to republish, to post on servers or to redistribute to lists, requires prior specific permission and/or a fee.

WICON 2007, October 22-24, Austin, USA

Copyright (C) 2007 978-963-9799-12-7

DOI 10.4108/pwsn.2007.2276 network protocol stack $^{[2]}$, MAC protocols is responsible for channel assignment and access control, while forwarding path construction and selection are usually left to routing protocols in the network layer. Therefore, most MAC protocols do not have the ability to choose receiving node independently, and it is difficult to achieve load balance. In communication, some nodes die too early for their fast energy consumption, which limits the network lifetime. In some serious cases, the network is even partitioned

In this paper, a forwarding election-based MAC protocol is put forward: the FE-MAC (Forwarding Election-based MAC) protocol. This protocol belongs to contention-based MAC protocols, which also has the routing capability of the network layer. Different from the traditional MAC protocols, data receiver is not specified by the upper routing protocol, but elected by neighboring nodes. The more energy a node remains, the more forwarding progress it provides, and the prior it will become a forwarding node. The advantages of FE-MAC protocol are: 1) it is a light-weight protocol. The control packet MAC protocol generated is used both for channel access control and routing decision making, which avoids the gathering of large amount of routing information, and improves the protocol efficiency; 2) Load balance is improved. The forwarding election mechanism makes nodes with more residual energy take more data forwarding task, the energy consumption is balanced, and the network lifetime is extended eventually.

The remainder of the paper is organized as follows. Section II reviews related work in the literature with reference to energy efficient MAC protocols and energy aware routing protocols for wireless sensor networks. Section III describes the detailed design of FE-MAC protocol, and a priority assignment algorithm is present to trade off the contradiction between routing efficiency and load balance. Section IV analyzes the performance of FEMAC and presents the simulation results. Finally, concluding remarks are drawn in section $\mathrm{V}$.

\section{RELATED WORK}

Constrained by energy and high related with application, it is hard to apply traditional wireless network protocols to WSN. Many proprietary network protocols are put forward for WSN.

S-MAC, T-MAC and B-MAC are contention based MAC protocols which adopt energy efficiency improvement as a major means to extend network lifetime. By reducing conflict, overhearing, idle listening and control overhead in 
communication, energy waste is reduced as much as possible. But, all nodes are in equal position when contending for the channel, nodes with less energy and nodes with more energy are equally treated, which speeds up energy consumption.

$\mathrm{BMA}^{[6]}$ TRAMA $^{[7]}$ and $\mathrm{LMAC}^{[8]}$ are schedule based MAC protocols, which can organize nodes to access channel without conflicts according to pre-determined TDMA schedule, nodes with no communication task will change into sleeping state, so as to reduce conflicts and idle listening. But there is large overhead in computing and allocating TDMA slots, it is hard to change schedule according to node's residual energy and network traffic, and it is difficult to realize load balance.

Being able to decide data's converging path, routing protocol can achieve energy savings from improvements in both energy efficiency and load balance. For example, SPIN ${ }^{[9]}$ protocol makes improvements for traditional flooding routing: nodes will negotiate before sending, and only send data to neighboring nodes which need them. GPSR ${ }^{[10]}$ is a geographical aware routing protocol., which adopts the rooting policy of greedy forwarding combined with Planar Perimeters Traversal Algorithm. Greedy forwarding algorithm is similar to mini-hop routing, if the node's transmitting power is fixed, the energy needed for forwarding one packet is minimum. In $\mathrm{LEACH}^{[11}$ protocol, cluster head is periodically chosen randomly in a round-robin mode, and energy consumption is balanced by means of averagely share network relaying. In [12], multiple independent paths are constructed from source node to destination node, to achieve load balance. In [13], multiple data gathering trees rooted from the converging node is formed, and energy consumption balance is achieved by making forwarding path to switch from one data gathering tree to another.

The above routing protocols make improvements in energy efficiency and load balance, which prominently extend network lifetime. But large amount of information is to be gathered for routing decision making such as routing establishment, cluster head election, multi-path construction and data gathering trees construction, which not only adds to energy consumption but also reduces routing efficiency. On the one hand, the communication from routing decision making is eventually imposed to MAC protocol, which adds to MAC protocol's control overhead and communication energy consumption. On the other hand, a period of time is needed to gather sufficient information, within which node state (position, residual energy, link quality etc.) might change, and as a result the time-effectiveness of the protocol is reduced and the failure probability and routing cost are increased.

\section{FE-MAC PROTOCOL DESIGN}

\subsection{Basic Scheme}

The FE-MAC protocol put forward by us, combined MAC and routing considerations, extends network lifetime from improvements both in energy efficiency and load balance. FEMAC adopts a RTS/CTS handshaking mechanism similar to 802.11 distributed coordination function (DCF). What different is that: the receiver's address is not included in RTS packet, and the only node to receive data finally is elected from neighboring nodes according to contending priority, which will be notified to the sender to construct forwarding path. The contending priority is related with node's residual energy and the forwarding progress. The above process is called Forwarding Election, by which a node with more residual energy will take more forwarding task, consequently, load balance and routing are achieved.

Definition 1 Given any two sensor nods $s_{i}$ and $s_{j}$ in WSN, if the Euclidean distance between them satisfies $d\left(s_{i}, s_{j}\right) \leq R_{c}$, then $s_{i}$ and $s_{j}$ are communication neighbors. While $R_{c}$ is the transmission radius of nodes, for simplicity, we suppose every node has the same $R_{c}$. All the communication neighbors of node $s_{j}$ are denoted by $\operatorname{Com} N(i)=\left\{s_{j} \mid d\left(s_{i}, s_{j}\right) \leq R_{c}, i \neq j\right\}$.

Definition 2 Given a sensor nods $s_{i}$ and the Sink node S, for any nodes $s_{j} \in \operatorname{Com} N(i)$, if $d\left(s_{j}, S\right) \leq d\left(s_{i}, S\right)$, then node $s_{j}$ is called $s_{i}$ 's forwarding neighbor. All the forwarding neighbors of node $s_{i}$ are denoted by $\operatorname{ForN}(i)=\left\{S_{j} \in \operatorname{ComN}(i) \mid d\left(s_{j}, S\right) \leq d\left(s_{i}, S\right)\right\}$, obviously, $\operatorname{For} N(i) \subseteq \operatorname{Com} N(i)$.

Definition 3 Given a sensor nods $s_{i}$ and the Sink node S, for any nodes $s_{j} \in \operatorname{ForN}(i), d_{f}=d\left(s_{i}, S\right)-d\left(s_{j}, S\right)$ is called the forwarding progress provided by $s_{j}$ for $s_{i}$.

\subsection{Periodic listen and sleep}

Each node goes to sleep for some time, and then wakes up and listens to see if any other node wants to talk to it. During sleep, the node turns off its radio, and set a timer to wake itself later. The duration of time for listening and sleeping is called a frame. For simplicity the length of a frame are the same for all nodes. Nodes choose their own listen/sleep schedules freely and exchange their schedules by broadcasting it to all its communication neighbors. FE-MAC adopts S-MAC's virtual clustering mechanism ${ }^{[]}$to guarantee the schedule synchronization between nodes. The virtual clustering scheme is easy to implement and make FE-MAC scalable and energy efficient.

During the startup phase of FE-MAC, each node will build a forwarding neighbor list FNL to record each communication neighbor's ID (address), the distance to Sink and the current residual energy etc. In node's communication activities, information in FNL will be a criterion to compute node's forwarding priority.

\subsection{Forwarding Election}

When a node is waken up, if there are data in buffer to send, it will first contend for the shared channel. When succeeding in obtain the channel, it becomes a sender and broadcast RTS request packet to all the communication neighbors. The forwarding neighbors having received the RTS packet, will decide the time to respond it according to local information and nodes with higher priority will send CTS packet earlier than other forwarding neighbors. When the sender receives the CTS packet, it will immediately send data packet to this node. In the head of the data packet, the address of the receiver is clearly specified, which acts as a CTS confirmation, and all the communication neighbors will update FNLs when receiving the data packet according to the head information, then go to sleep until the transfer is finished. After the forwarding node receives a complete data packet, it will send an ACK packet to confirm, which denotes the completion of a data transfer. Afterwards, all the nodes continue listening to the channel, and the above process is repeated, until the active period ends and all the nodes go to sleep. The basic working principle of forwarding election is depicted in Figure 1. 


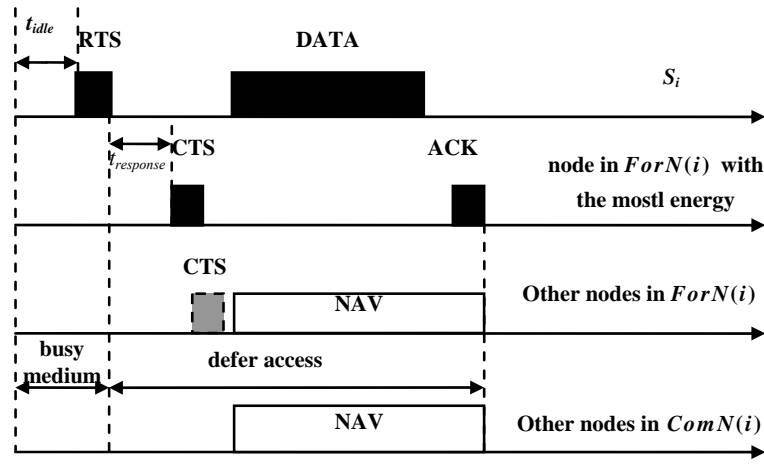

Fig. 1 Working principle of FE-MAC protocol

In FE-MAC protocol, a node has 4 states: LISTEN, RECEIVE, CONTEND and SLEEP. Under each state, the working procedure and state switch process is given in Figure 2, where $t_{\text {idle }}$ denotes the idle waiting time, $t_{\text {back }}$ denotes the random back-off time, and $t_{c t s}, t_{\text {data }}$ and $t_{a c k}$ denote the longest interval to wait for CTS, DATA and ACK packets respectively.

Noted that, after the sender node $s_{i}$ sends a RTS request packet, as $s_{i}{ }^{\text {'s }}$ forwarding neighbors are not definitely communication neighbors, multiple CTS responses might collide each other at the receiver side. FE-MAC uses Collision Resolution Algorithm to solve this problem. If the sending node $s_{i}$ receives an irresolvable signal, collision is deemed to occur among multiple CTS packets. At this moment, node $s_{i}$ will send a RPT packet to trigger the Collision Resolution Algorithm. The forwarding neighbors receiving the RPT packet will judge according to the following rules:

Rule 1: All forwarding neighbors who did not transmit CTS packet drop out and waiting for ACTS packet from the sender;

Rule 2: Nodes who have send CTS before decide with probability 0.5 whether or not to retransmit CTS packet during the next $t_{\text {conflict }}$ time. Three events are possible: 1 ) only one node sends, transmitter starts packet transmission and all others drop; 2) more than one CTS are send, transmitter sends a RPT message, those who did not send drop out, those involved in the collision decide whether or not to continue as before, until the collision is resolved; 3) no CTS is heard, a RPT message is send by the transmitter, and all nodes who did not drop out decide again independently whether to continue as before.

Rule 3: The transmitter send a QIT packet if the RPT packet have send more than $N_{\text {MaxRpt }}$ times, and give up current data transmission. After receive the QIT packet, all forwarding neighbors return to LISTEN state and listen the channel until the active time is expired.

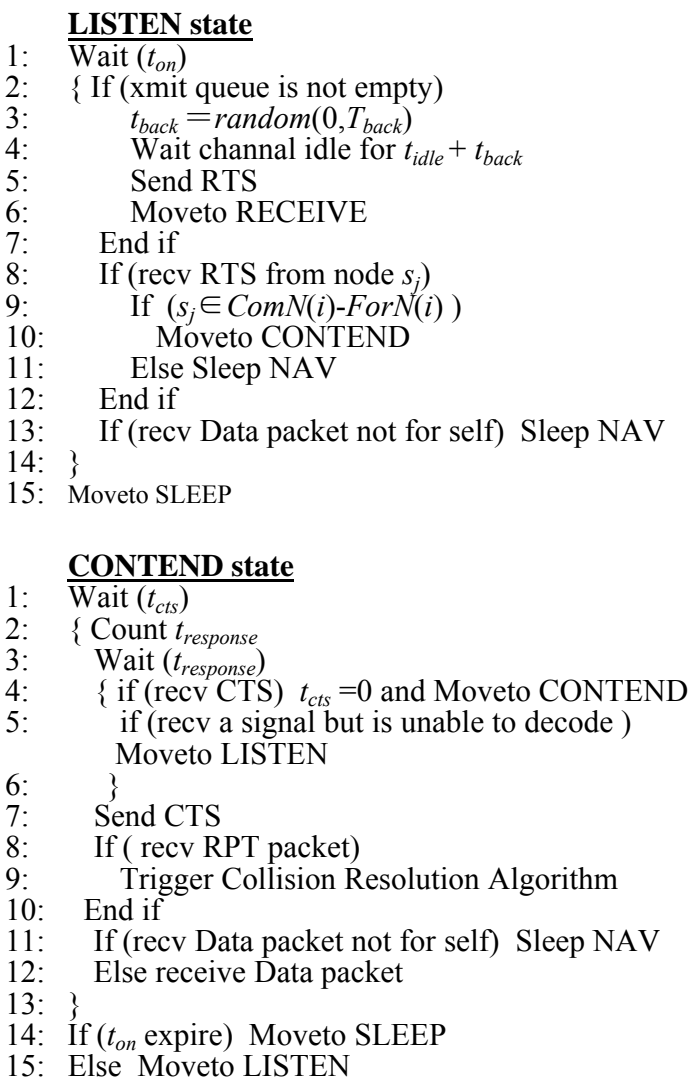

\section{CONTEND state}

Wait $\left(t_{c t s}\right)$

$\left\{\right.$ Count $t_{\text {response }}$

Wait ( $\left.t_{\text {response }}\right)$

$\left\{\right.$ if (recv CTS) $t_{c t s}=0$ and Moveto CONTEND

if (recv a signal but is unable to decode ) Moveto LISTEN

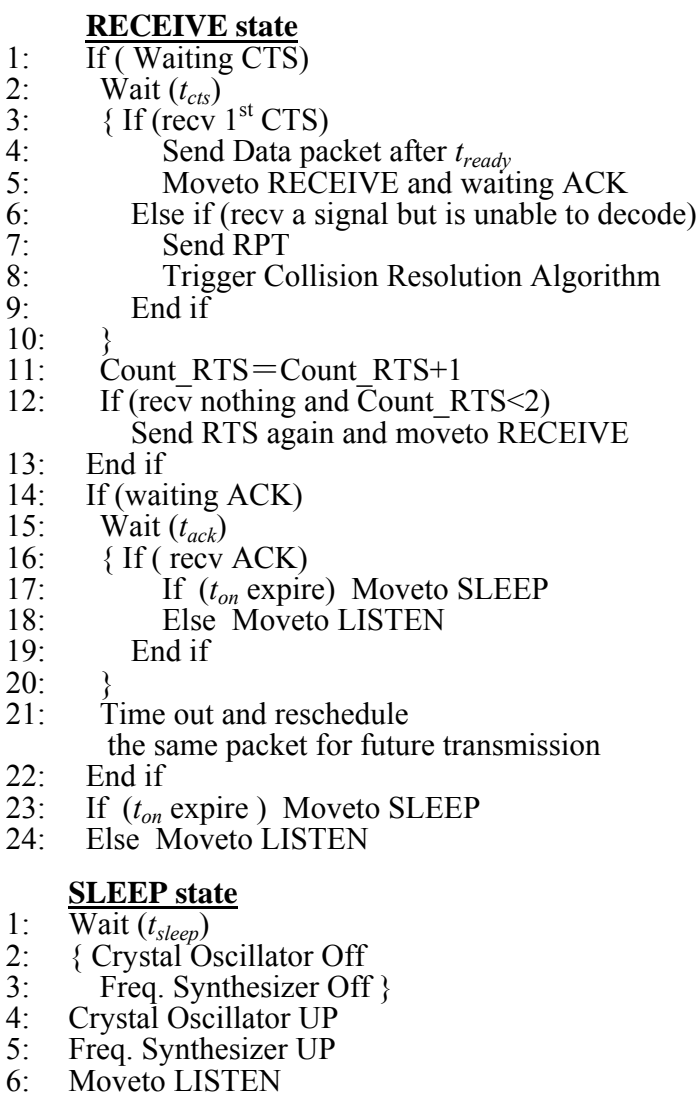

Fig.2 Pseudocode for the different states and state transitions in FE-MAC 
Property 1 Forwarding election will not generate loop.

Proof. Line 10 of LISTEN state is the only entrance to CONTEND state, line 9 guarantees that only forwarding neighbors will participate in the contention, therefore forwarding node will be definitely generated among forwarding neighbors. It is known from Definition 2 that, forwarding data will only be received by those nodes that are nearer to Sink, therefore, there is no loop in forwarding path.

Property 2 FE-MAC protocol will not lead to deadlock.

Proof. Under the state of LISTEN, nodes will not wait infinitely. Line 5 and line 10 guarantee that node will transit to RECEIVE state and CONTEND state, and line 1 and line 15 guarantee that node will transit to SLEEP state no longer than $t_{\text {on }}$; Under the state of RECEIVE, line 2 and line 15 guarantee that nodes will not infinitely wait for CTS or ACK packet. A complete data transfer will not be confirmed (line 18) until an ACK packet is eventually received. Rule 3 in Collision Resolution Algorithm guarantee that the execution time of the algorithm will be shorter than $N_{\text {MaxRpt }} \times t_{\text {conflict }}$; Line 1,14 and 15 within CONTEND state guarantee that the time a node keeping in CONTEND state will be no longer than $t_{c t s}$; and finally, line 1 and line 6 within SLEEP state will make a node return to LISTEN state after a period of tsleep. To sum up, a node will not stay in any state for ever, therefore FE-MAC protocol will not lead to deadlock.

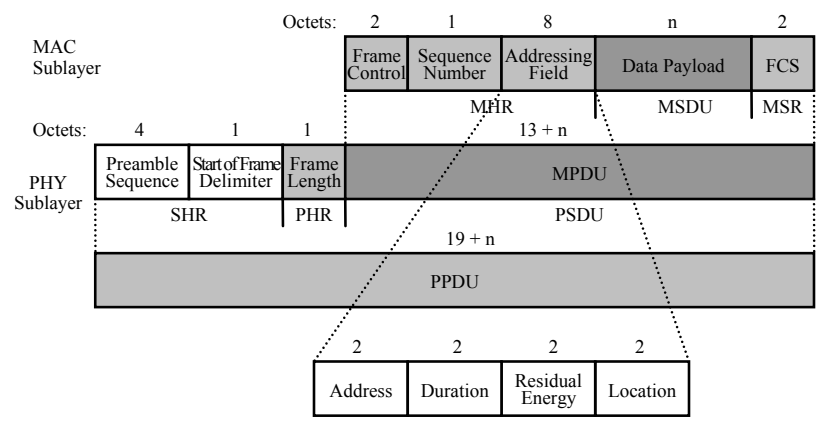

Fig. 3 Frame structure of FE-MAC protocol

Nodes carry the information about location and residual energy in RTS, CTS and ACK packets and data packet's head, so any communication node can update its forwarding neighbor list FNL accordingly. In the Priority Allocation Algorithm introduced later, forwarding neighbors will compute the response time $t_{\text {response }}$ according to FNL's information. The packet structure of FE-MAC protocol is given in Figure3. Compared with 802.15.4's MAC protocol, the original address field with a maximal length of 20 Bytes is shortened to 8 Bytes, three fields Duaration, Residual Energy and Location are added, and the other control fields remain unchanged.

\subsection{Priority Allocation Algorithm and response time computing}

From the aspect of maximizing network lifetime, many performance parameters are related to a node's energy efficiency, such as residual energy, forwarding progress, length of buffer queue and channel quality etc. To balance each node's energy consumption and minimize the forwarding hop, we choose residual energy and forwarding progress as criteria to priority.
Deem that, the maximum and minimum residual energy of all the forwarding neighbors are $e_{\max }$ and $e_{\min }$ respectively, forwarding neighbor $s_{i}$ 's current residual energy is $e_{i}$, and the distances between sending node and node $s_{i}$ to Sink are $d_{s}$ and $d_{i}$ respectively, then node $s_{i}$ 's response priority is:

$$
\chi_{i}=\alpha\left(\frac{e_{\max }-e_{i}}{e_{\max }-e_{\text {min }}}\right)+(1-\alpha)\left(1-\frac{d_{s}-d_{i}}{R_{c}}\right) \quad(0 \leq \alpha \leq 1)
$$

Where parameter $\alpha$ is used to adjust the weights of residual energy and forwarding progress in response priority, and guarantee that $0 \leq \chi_{i} \leq 1$, we will discuss $\alpha$ 's value in experiments. and in Equation (1) are obtained by sending node according to its local FNL, $e_{\max }$ and $e_{\min }$ are broadcasted to communication neighbors carried in RTS packet. That is, $e_{\max }$ and $e_{\text {min }}$ in Equation (1) are from sending node's FNL, which is also an estimated value. Though it is not a precise value, we argue that they are only references for nodes, and the existence of estimation error will not affect the correctness of the protocol. As the residual energy in FNL is conservatively estimated, updated only when change confirmedly occurs, and will become less and less, the residual energy of the current forwarding neighbors will not surpass $e_{\max }$ and $e_{\max }$ still holds; The node with more residual energy has a higher probability of becoming the forwarding node, once elected, it will restrain the other nodes' election contention, therefore, the error from $e_{\min }$ also has a small impact on the protocol. Besides, FE-MAC aims at balanced energy consumption among nodes and avoids the overuse and over-idlesse of a single node, which is beneficial to FNL's prompt update and revision, and reduces the accumulation of estimation errors.

After the forwarding neighbor receives the RTS packet, it can not send CTS packet until at least $t_{\text {switch }}$ time has passed. The response time depends on the node's response priority and should not surpass the response delay $t_{c t s}$, the response time $t_{\text {response }}$ is computed as follows:

$$
t_{\text {response }}=\left(t_{\text {switch }}-t_{c t s}\right) \sin \left(\frac{\pi}{2} \chi_{i}\right)+t_{c t s}
$$

Where the sine function is to reduce collision among nodes with an approximately highest priority, utilizing sine function's characteristics of being a protruding function. From Equation (2), it can be seen that $t_{\text {switch }} \leq t_{\text {response }} \leq t_{c t s}$, and the higher response priority a forwarding neighbor has, the shorter it waits, and the earlier it sends CTS.

Table1 Values for various time parameter

\begin{tabular}{|c|c||c|c|}
\hline$t_{\text {idle }}$ & $128 \mu \mathrm{s}$ & $t_{\text {response }}$ & $64 \mu \mathrm{s} \sim 1.6 \mathrm{~ms}$ \\
\hline$t_{\text {back }}$ & $0 \sim 2.05 \mathrm{~ms}$ & $T A$ & $5.6 \mathrm{~ms}$ \\
\hline$t_{\text {cts }}$ & $1.6 \mathrm{~ms}$ & $t_{T A}$ & $4 \mathrm{~ms}$ \\
\hline$t_{\text {data }}$ & $192 \mu \mathrm{s}$ & $t_{\text {ready }}$ & $64 \mu \mathrm{s}$ \\
\hline$t_{A C K}$ & $192 \mu \mathrm{s}$ & $t_{\text {conflict }}$ & $64 \mu \mathrm{s}$ \\
\hline$t_{\text {on }}$ & $50 \mathrm{~ms}$ & $t_{\text {switch }}$ & $64 \mu \mathrm{s}$ \\
\hline
\end{tabular}

In Table 1, according to the physical characteristics of sensor nodes MICAz and Tmote Sky[14] developed by UC Berkeley, values for various time parameters related to FE-MAC protocol 
are discussed. The above nodes both use $2.4 \mathrm{GHz} \mathrm{CC} 2420$ radio, which can generate a data transfer rate of $250 \mathrm{kbps}$. For simplicity, deem that the length of the maximum effective data load is 128Bytes, the length of RTS, CTS, RPT packet and the data packet head are all 13Bytes (as is depicted in Figure 3), and the length of ACK packet is 7Bytes $(2+1+2+2)$. For the convenience of later comparison, a duty-cycle of $10 \%$ is adopted in Table 1 same as S-MAC ptotocol's.

\section{EXPERIMENTS AND ANALYSIS 4.1 Parameter settings}

We simulate S-MAC, T-MAC and FE-MAC protocol using ns2 simulator, and make comparison between them. Besides the time parameters given in Table 1, the other parameters are given in Table 2 .

Since S-MAC and T-MAC do not have the ability of routing, we use GPSR protocol as their upper routing protocol, i.e., the neighboring node that is nearest to the Sink is always chosen as forwarding node. Meanwhile, for the representativeness of the comparison, we deem that the routing process of the GPSR protocol is costless. Considering that the three protocols adopt the same sleeping schedule and clock synchronization techniques, to simplify the experiments, we deem that only one virtual cluster is formed in the network and the duty-cycle is the same. In the following, without specification, the results are all averages of 100 simulation runnings under the same condition.

Table2 Simulation Parameters

\begin{tabular}{|l|c||l|c|}
\hline radio & CC2400 & Tx power & $55 \mathrm{~mW}$ \\
\hline Data rate & $250 \mathrm{kbps}$ & Idle power & $1.88 \mathrm{~mW}$ \\
\hline Rating voltage & $3 \mathrm{~V}$ & Sleeping power & $0.54 \mathrm{~mW}$ \\
\hline Comm. radius & $30 \mathrm{~m}$ & Duty cycle & 0.1 \\
\hline Initial energy & $3 \mathrm{Ah}$ & Sleeping time & $450 \mathrm{~ms}$ \\
\hline Rx power & $65 \mathrm{~mW}$ & Frame length & $500 \mathrm{~ms}$ \\
\hline
\end{tabular}

\subsection{Results and analysis}

We compare the network lifetime of S-MAC, T-MAC and FEMAC protocol with different node number (density). The network lifetime is defined as the interval between the completing of network configuration and the time when the first node uses up its energy. For S-MAC and T-MAC protocol, we first compute GPSR routing with centralized algorithm, and deem that all the nodes have obtained the routing information for the next hop, as is shown in Figure 4. To generate data traffic, the node will gather the environment information once a fixed period of time, and each time $1 \mathrm{~kb}$ sensing data is generated. Since all the nodes will gather data, sensing power consumption is ignored. As the connecting probability in the network with a scale of less than 200 nodes is not high, we only compare network with a scale of more than 200 nodes. And in FE-MAC protocol, $\alpha$ is set to 0.5 , and the data transfer rate is set to 4 packets/s.

From the results in Figure 5, we can see that, though we deem that there is no routing overhead in the upper GSPR protocol of SMAC and T-MAC, with different node number (density), FEMAC protocol still achieves longer network lifetime than S-MAC and T-MAC. And the more the number of nodes is, the longer FE-
MAC protocol's lifetime is. For a network with 200 nodes in scale, FE-MAC can achieve a lifetime of 319.7 days, which is increased by $469 \%$ and $204 \%$ as compared with S-MAC and T-MAC's lifetime (56.2 days and 105 days). This is because that S-MAC and T-MAC protocol bind static routing, nodes near to Sink soon use up their energy for taking too much forwarding task, while a large number of nodes, especially those edge nodes still have sufficient energy. In FE-MAC protocol, network lifetime is extended from improvements in both energy efficiency and load balance, the converging path for data forwarding will adjust constantly according to the node's current residual energy, which makes node energy consumption more balanced. Though it is still the nodes near to Sink die first, FE-MAC well balances these nodes' energy consumption, and extends the network lifetime to a large extent.

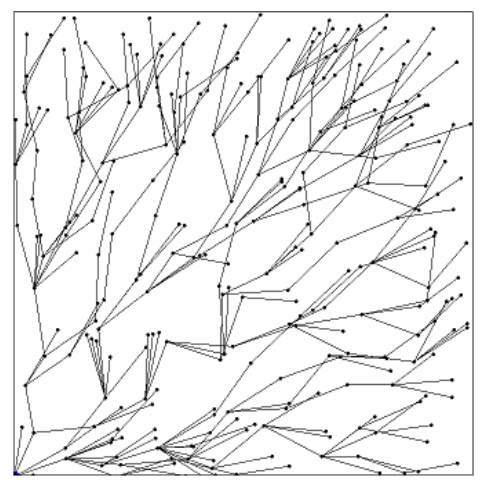

Fig. 4 Topology of 300 nodes in a $200 * 200$ network

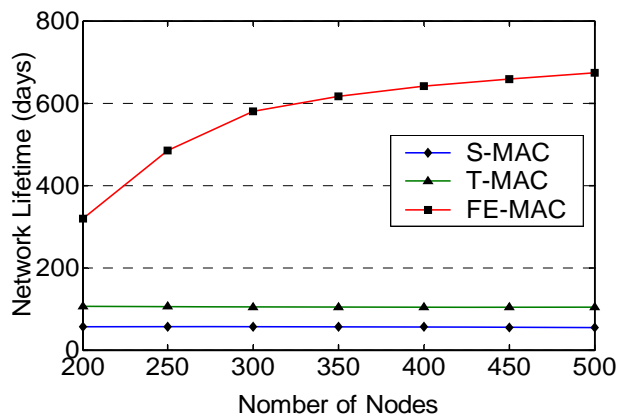

Fig. 5 Network Lifetime vs. Number of nodes

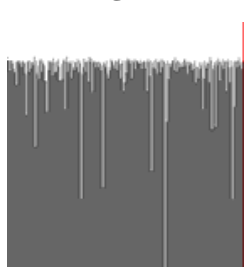

(a) S-MAC

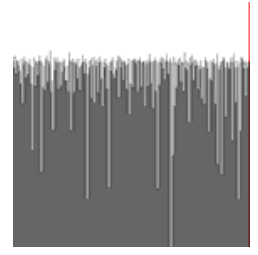

(b) T-MAC

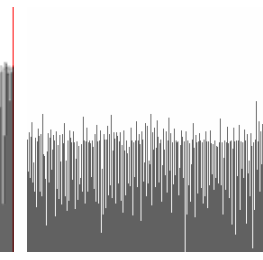

(c) FE-MAC
Fig. 6 Final residual energy of nodes

To better know a node's energy variation, we track its residual energy in experiments. In Figure 6, the final residual energy of SMAC, T-MAC and FE-MAC's nodes are depicted when the network lifetime ends, and it can be seen that the differences in residual energy between FE-MAC nodes are the smallest, which is 
the result of load balance. Note that, in our experiments the overhead in GPSR is not considered, therefore in real application, FE-MAC will obtain even longer network lifetime.

In the priority computing equation (3), parameter decides the weights of residual energy and forwarding progress in response priority. To observe the protocol's whole performance, we set in the previous experiments, but this setting is not the optimum for different network environment. To observe the impact of on FEMAC, we compare the relation between the network lifetime and parameter in networks of different scale, as is shown in Figure 7. The results show that the different value of lay a great impact on the network lifetime. For networks in different scale, there is an optimum value for, which is within the interval of $(0.5,0.9)$. This also shows that the longest network lifetime cannot be obtained if the response priority is decided only according to forwarding progress or residual energy, there should be a tradeoff. The results show that, the weight of residual energy in response priority should be higher than forwarding progress.

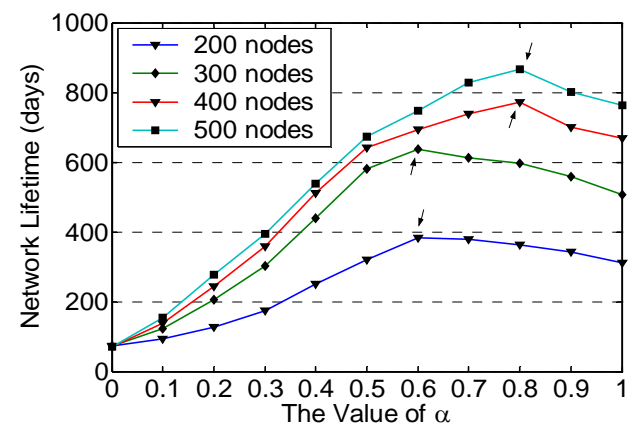

Fig. 7 Network Lifetime vs. parameter $\alpha$

\section{CONCLUSION}

In this paper, a forwarding election based FE-MAC protocol is put forward, which aims at extends network lifetime from improvements in both energy efficiency and load balance. What's different from the traditional MAC protocols is that, FE-MAC protocol also has the routing capability of the network layer. FEMAC protocol adopts the framework of periodic listen and sleep scheduling from S-MAC, and inherits its scalability and energy efficiency. Meanwhile, a forwarding election mechanism based on priority is adopted, which organizes nodes to contend forwarding right according to the current residual energy, and makes nodes with more residual energy take more forwarding tasks to achieve load balance. The experiments show that, compared with S-MAC and T-MAC protocol, FE-MAC can prominently extend the network lifetime of network in different scale, and the larger the number of node is, the longer the network lifetime is. It is more suitable to configure FE-MAC in large scale WSNs.

\section{REFERENCES}

[1] Bowman, B., Debray, S. K., and Peterson, L. L. Reasoning about naming systems. ACM Trans. Program. Lang. Syst., 15, 5 (Nov. 1993), 795-825.
[2] I F Akyildiz, W Su, Y Sankarasubramaniam. Wireless sensor networks: A survey [J]. Computer Networks, 2002, 38(4) 393-422.

[3] Pister k, Hohlt B, Jeong J, Doherty L, Vainio J P. Ivy - A sensor network infrastructure. 2003. http://www-bsac.eecs. berkeley.edu/projectes/ivy.

[4] W. Ye, J. Heidemann, and D. Estrin. An energy-efficient mac protocol for wireless sensor networks. In In Proceedings of the 21st International Annual Joint Conference of the IEEE Computer and Communications Societies (INFOCOM 2002), June 2002.

[5] T. van Dam and K. Langendoen. An adaptive energy efficient mac protocol for wireless sensor networks. In 1st ACM Conference on Embedded Networked Sensor Systems (SenSys), 2003, 171-180.

[6] J. Polastre, J. Hill, and D. Culler. Versatile low power media access for wireless sensor networks. In The Second ACM Conference on Embedded Networked Sensor Systems (SenSys), November 2004, 95-107.

[7] J. Li and G. Lazarou. A bit-map-assisted energy-efficient MAC scheme for wireless sensor networks. In 3rd Int. Symp. on Information Processing in Sensor Networks (IPSN04), Berkeley, April 2004, 55-60.

[8] Venkatesh Rajendran, Katia Obraczka, and J.J. GarciaLunaAceves, Energy-Efficient, Collision-Free Medium Access Control for Wireless Sensor Networks, 1st ACM Conference on Embedded Networked Sensor Systems (SenSys2003), November 2003.

[9] L. van Hoesel and P. Havinga. A lightweight medium access protocol (LMAC) for wireless sensor networks. In 1st Int. Workshop on Networked Sensing Systems (INSS 2004), Tokyo, Japan, June 2004.

[10] Kulik J, Heinzelman WR, Balakrishnan H. Negotiation based protocols for disseminatinginformation in wireless sensor networks. Wireless Networks, 2002,8(2-3):169-185.

[11] Karp B, Kung H. GPSR: Greedy perimeter stateless routing for wireless networks. In: Proc. of the 6th Annual Int'l Conf. on Mobile Computing and Networking. Boston: ACM Press, 2000. 243-254.

[12] Heinzelman W, Chandrakasan A, Balakrishnan H. Energyefficient communication protocol for wireless microsensor networks. In: Proc. of the 33rd Annual Hawaii Int'l Conf. on System Sciences. Maui: IEEE Computer Society, 2000. 3005-3014.

[13] Ganesan D, Govindan R, Shenker S, Estrin D. Highlyresilient, energy-efficient multipath routing in wireless sensor networks. Mobile Computing and Communications Review (MC2R). 2002, 1(2).

[14] Zhang Qing, Xie Zhipeng, Ling Bo, Sun Weiwei, Shi Bole. A Maximum Lifetime Data Gathering Algorithm for Wireless Sensor Networks [J]. Journal of Software, 2005, 1946-1957.

[15] MoteIV Corporation, Telos Revision A Mote, http://www.moteiv.com, February 2006. 